\title{
CUMBRES INDÍGENAS: POLÍTICA Y DIPLOMACIA ANCESTRAL EN AMÉRICA LATINA
}

Nasly Cruz Chavarro

Gabriel Andrés Arévalo Robles

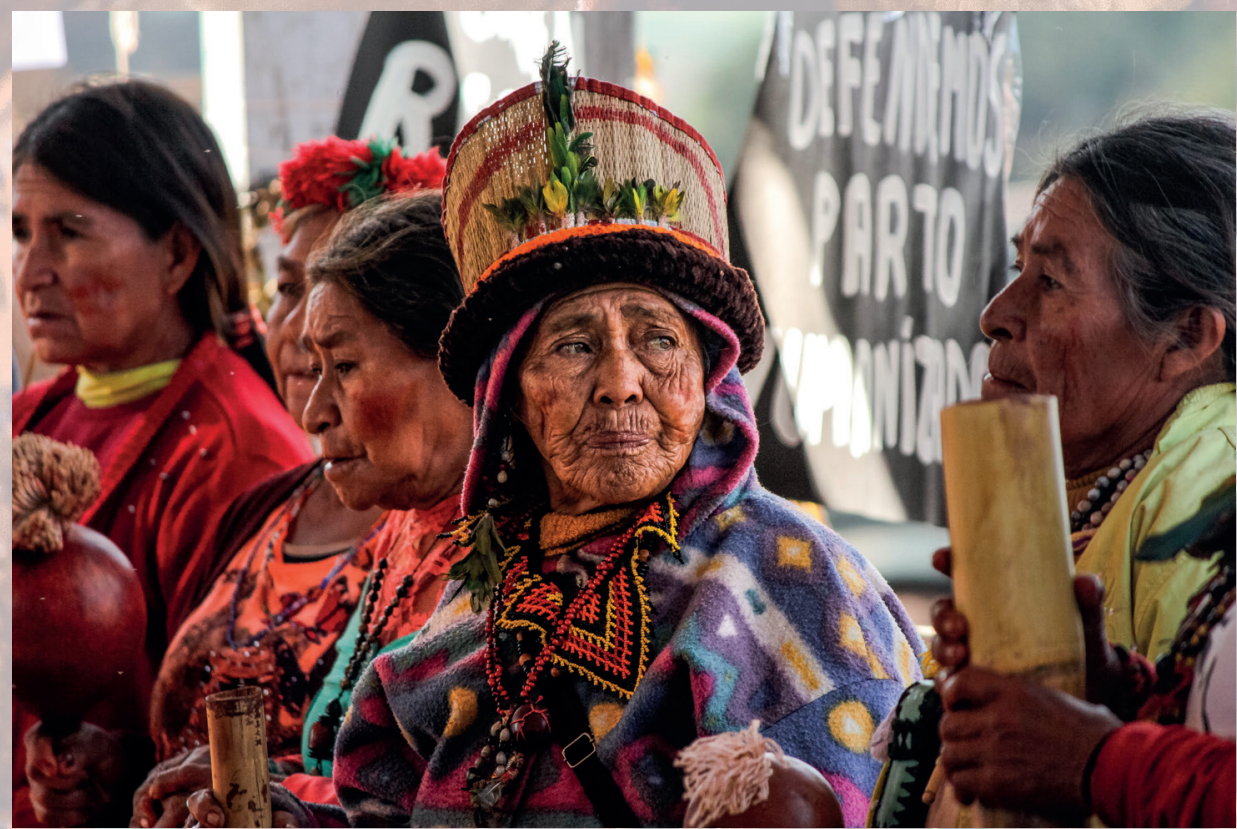





\title{
CUMBRES INDÍGENAS: POLÍTICA Y DIPLOMACIA ANCESTRAL EN AMÉRICA LATINA*
}

\author{
Nasly Cruz Chavarro \\ Gabriel Andrés Arévalo Robles \\ Universidad Nacional Autónoma de México \\ Universidad Católica de Colombia
}

\section{Resumen}

La diplomacia indígena es una práctica social y política recurrente de representantes indígenas, para mediar, incidir y negociar sus apuestas políticas en escenarios y organismos internacionales y regionales. Una de sus principales y más novedosas dimensiones son los encuentros y cumbres diplomáticas indígenas, que se convirtieron en estrategias de negociación ancestral para la construcción de horizontes políticos continentales. El presente artículo expone los principales hallazgos de la siguiente pregunta de investigación: ¿cuáles han sido las dimensiones políticas, funcionales y simbólico/culturales de la diplomacia indígena de los encuentros y cumbres ancestrales realizados en América Latina en los últimos treinta años? Los principales hallazgos fueron: a) el horizonte político indígena latinoamericano evolucionó hacia la concepción de la 'toma del poder'; b) el cambio de milenio se concibió como una señal del tiempo cíclico para la recuperación del territorio ancestral, tomando como referencia la autodeterminación; c) la espiritualidad es la inspiración y guía de las luchas ancestrales; d) la lucha de las mujeres indígenas por la apertura de espacios de participación continental. Cada una de estas respuestas será desarrollada a lo largo del presente texto.

Palabras clave: diplomacia indígena, cumbres indígenas, política indígena latinoamericana, autodeterminación, participación de mujeres indígenas.

\footnotetext{
La investigación pertenece a la línea de investigación "Fundamentación e implementación de los derechos humanos", del grupo de investigación Persona, Instituciones y Exigencias de Justicia, reconocido y categorizado como tipo Al por el Ministerio de Ciencia, Tecnología e Innovación (Minciencias) y registrado con el código COL 0120899, vinculado al Centro de Investigaciones Sociojurídicas (CISJUC), adscrito y financiado por la Facultad de Derecho de la Universidad Católica de Colombia.
} 
Los autores: Nasly Cruz Chavarro, Candidata a magíster en Estudios Latinoamericanos de la Universidad Nacional Autónoma de México (UNAM). Licenciada en Ciencias Sociales de la Universidad Distrital Francisco José de Caldas. Correo electrónico: naslycruz2903@gmail.com

Gabriel Andrés Arévalo Robles, Doctor en Estudios Internacionales de la Universidad del País Vasco/ Euskal Herriko Unibertsitatea, UPV/EHU. Máster en Estudios Internacionales de la UPV/EHU. Abogado de la Universidad Libre. Sociólogo de la Universidad Nacional de Colombia. Docente investigador de la Facultad de Derecho, Universidad Católica de Colombia. Grupo de investigación Personas, Instituciones y Exigencias de Justicia, categoría de Colciencias Al. Correo electrónico: gaarevalo@ucatolica.edu.co

Recibido: 13 de junio de 2020; evaluado: 20 de agosto de 2020; aceptado: 8 de octubre de 2020. 


\title{
INDIGENOUS SUMMITS: POLITICS AND ANCESTRAL DIPLOMACY IN LATIN AMERICA
}

\author{
Nasly Cruz Chavarro \\ Gabriel Andrés Arévalo Robles \\ Universidad Nacional Autónoma de México \\ Universidad Católica de Colombia
}

\begin{abstract}
Indigenous diplomacy is a recurring social and political practice of indigenous representatives to mediate, influence, and negotiate their political stakes in international and regional organizations. One of its main and most innovative dimensions is the indigenous diplomatic summits that have become ancestral negotiation strategies for the construction of continental political horizons. This article presents the main findings of the following research question: what have been the political, functional, and symbolic/cultural dimensions of the indigenous diplomacy of the Latin American Ancestral Summits held in the last thirty years? The main findings include the following: a) the Latin American indigenous political horizon evolved towards the concept of 'taking power;' b) the turn of the millennium was conceived as a sign of the cyclical time for the recovery of ancestral territory, using the principle of self-determination as a reference; c) spirituality is the inspiration and guide of ancestral struggles; d) the struggle of indigenous women to open spaces for continental participation. Each of these findings is developed throughout this text.
\end{abstract}

Keywords: indigenous diplomacy, indigenous summits, Latin American indigenous politics, selfdetermination, participation of indigenous women. 
Authors: Nasly Cruz Chavarro, Master's candidate in Latin American Studies from the National Autonomous University of Mexico (UNAM). BA in social sciences from the Francisco José de Caldas District University. Email: naslycruz2903@gmail.com.

Gabriel Andrés Arévalo Robles, Doctor in International Studies from the Basque Country University/ Euskal Herriko Unibertsitatea (UPV/EHU). Master's in International Studies from UPV/EHU. Lawyer from the Free University, sociologist from the National University of Colombia. Research professor in the Catholic University of Colombia Law School. Persons, Institutions, and Justice Demands research group, Colciencias category A. Email: gaarevalo@ucatolica.edu.co

Received: June 13, 2020; evaluated: August 20, 2020; accepted: October 8, 2020. 


\title{
REUNIÕES DE CÚPULA INDÍGENAS: POLÍTICA E DIPLOMACIA ANCESTRAL NA AMÉRICA LATINA
}

\author{
Nasly Cruz Chavarro \\ Gabriel Andrés Arévalo Robles \\ Universidad Nacional Autónoma de México \\ Universidad Católica de Colombia
}

\section{Resumo}

A "diplomacia indígena" é uma prática social e política recorrente de representantes indígenas para mediar, incidir e negociar suas apostas políticas em cenários e organismos internacionais e regionais. Uma de suas dimensões principais e mais inovadoras são os "encontros e reuniões de cúpula diplomáticas indígenas", que se converteram em estratégias de negociação ancestral para a construção de horizontes políticos continentais. O presente artigo expõe as principais descobertas da seguinte pergunta levantada na pesquisa: quais foram as dimensões políticas, funcionais e simbólico/culturais da diplomacia indígena dos encontros e reuniões de cúpula ancestrais realizados na América Latina nos últimos 30 anos? As principais descobertas foram: a) o horizonte político indígena latino-americano evoluiu em direção à concepção da "tomada de poder"; b) a mudança de milênio foi concebida como um sinal do tempo cíclico para a recuperação do território ancestral, que tomou como referência a autodeterminação; c) a espiritualidade é inspiração e guia das lutas ancestrais; d) a luta das mulheres indígenas pela abertura de espaços de participação continental. Cada uma dessas respostas será desenvolvida ao longo do presente texto.

Palavras-chave: diplomacia indígena, reuniões de cúpula indígenas, política indígena latino-americana, autodeterminação, participação de mulheres indígenas. 
Os autores: Nasly Cruz Chavarro, Mestranda em Estudos Latino-americanos pela Universidade Nacional Autônoma do México (UNAM). Licenciada em Ciências Sociais pela Universidade Distrital Francisco José de Caldas. E-mail:

Gabriel Andrés Arévalo Robles, Doutor em Estudos Internacionais pela Universidade do País Basco/ Euskal Herriko Unibertsitatea (UPV/EHU). Mestre em Estudos Internacionais pela UPV/EHU. Advogado pela Universidade Libre. Sociólogo pela Universidade Nacional da Colômbia. Docente e pesquisador da Faculdade de Direito, Universidade Católica da Colômbia. Grupo de pesquisa "Personas, Instituciones y Exigencias de Justicia", categoria Al no Colciencias. E-mail:

Recebido: 13 de junho de 2020; avaliado: 20 de agosto de 2020; aceito: 8 de outubro de 2020. 


\section{Introducción}

El presente artículo pretende comprender un particular fenómeno de la globalización contrahegemónica, conocido como diplomacia indígena, en la especial dimensión de los encuentros y cumbres continentales de pueblos, naciones y mujeres indígenas de los últimos treinta años en Latinoamérica. La diplomacia indígena es entendida en este texto con un doble significado: por un lado, es un campo semántico de discusión sobre la noción clásica de la diplomacia; por el otro, es la descripción de una práctica indígena en la que líderes y representantes indígenas median, inciden y negocian sus apuestas políticas con organismos y organizaciones políticas en un sinnúmero de temas y situaciones ${ }^{1}$.

Lo diplomático y lo indígena deben ser entendidos desde ciertas aclaraciones. La palabra diplomacia no debe ser comprendida exclusivamente como una actividad estatal; por el contrario, es una práctica humana usual e histórica que refiere a la alteridad cuando entidades diferentes (pueblos, naciones, grupos, comunidades) se encuentran y buscan gestionar su diferencia por medio de normas y procedimientos constantes o ad hoc, como ha sido confirmado en diferentes épocas de la historia ${ }^{2}$. Por su parte, indígena, a pesar de ser una noción usada por los colonizadores, fue recreada políticamente para enfrentar las relaciones de dominación y la explotación ${ }^{3}$.

La práctica diplomática indígena tiene diferentes expresiones: a) la diplomacia indígena internacionalizada, que se despliega en las organizaciones internacionales, principalmente - pero no exclusivamente - en la Organización de las Naciones Unidas, a partir de la década de los ochenta; b) la diplomacia indígena enraizada, que es la actividad diplomática desarrollada en razón del territorio — necesidades, problemas y retos políticos, económicos y/o culturales_- y en la que usualmente participan representantes y líderes indígenas de diferentes países y regiones, como en caso del zapatismo o de la triple alianza de alcaldes aymaras en Chile, Perú y

Gabriel Andrés Arévalo Robles, "La diplomacia indígena: un enfoque transdiplomático", Si Somos americanos, vol. 1, n. ${ }^{\circ}$ 17. (2017): 141-169. https://dx.doi.org/10.4067/S0719-09482017000100141

2 James Der Derian, On diplomacy: A genealogy of western estrangement (Oxford: Blackwell Publishers, 1987); Costas Constantinou y James Der Derian, coord., Sustainable diplomacies (New York: Palgrave MacMillan, 2010); Constantinou Costas, "On homo-diplomacy", Space and Culture, vol. 9, n. ${ }^{\circ} 4$ (2006): 351-364; Constantinou Costas, On the way to diplomacy (Minneapolis: University of Minnesota Press, 1996); Noé Cornago, Plural diplomacies: Normative predicaments and functional imperatives (Boston: Martinus Nijhoff Publishers, 2013); Gabriel Andrés Arévalo Robles, "Repensar la historia diplomática: diálogos, ausencias y retos para el entendimiento de la alteridad histórica mundial", Relaciones Internacionales, n. 37 (mayo, 2018): 121-141. doi: http://dx.doi.org/ 10.15366/relacionesinternacionales2018.37.00

Fausto Reinaga, Tesis india (La Paz: Ediciones PIB, 1971). 
Bolivia; y c) la diplomacia de la complementariedad, que es la practicada entre pueblos indígenas para superar y enfrentar los retos que suponen las asimétricas relaciones históricas a las que se han visto sometidos. Esta última dimensión será el objeto de la presente investigación.

Antes de la Conquista, los 'pueblos indígenas' interactuaron por medio de procesos de ancestral diplomacia; crearon vínculos a través de las fronteras de su comunidad, conformaron y establecieron inmediatos o permanentes sistemas de comunicación e intercambio entre diferentes pueblos y naciones a lo largo de sus territorios ${ }^{4}$. La diplomacia indígena no desapareció en la Colonia; por el contrario, las relaciones diplomáticas entre naciones indígenas y colonizadores fue permanente, como en el caso de la figura del Parlamento en el actual Chile. Tampoco se diluyó durante la formación de las repúblicas del siglo XIX; siguieron existiendo, aunque tenuemente, relaciones con los nuevos gobiernos. Sin embargo, la interacción indígena en las nuevas repúblicas fue reprimida fuertemente por los gobiernos criollos que pretendieron absorberla en su nación civilizatoria ${ }^{5}$.

En las últimas décadas del siglo XX hubo un resurgir de la fuerza indígena. La reinscripción histórica de la identidad indígena recreó y fortaleció las relaciones étnico-políticas transfronterizas. El relato étnico identitario promovió reivindicaciones y reclamos en escenarios internacionales y regionales sobre temas asociados con la desigualdad, y ganaron apoyos y alianzas por medio de la autopista global ${ }^{6}$. Como consecuencia de esta actividad diplomática transfronteriza, se desarrolló una identidad étnica global determinante para alcanzar logros jurídico-políticos internacionales, discutir con las instituciones políticas internacionales y, de regreso, enfrentar a "sus" Estados. Los y las representantes indígenas se articularon a fuerzas transnacionales, como organizaciones no gubernamentales, iglesias y organizaciones académicas para mover su actividad diplomática, motivados por ideas y valores, así como con

Jennings Francis y William Fenton, The history and culture of Iroquois diplomacy: An interdisciplinary guide to the treaties of the six nations and their league (Syracuse University Press, 1995); Tom Arne Midtrød, The memory of all ancient customs: Native American diplomacy in the colonial Hudson Valley (Cornell: University Press, 2012); Richard Aquila, The Iroquois Restoration: Iroquois diplomacy on the colonial frontier, 1701-1754 (Nebraska Press, 1997).

5 Abelardo Levaggi, Diplomacia Hispano-Indígena en las fronteras de América: Historia de los tratados entre la monarquía española y las comunidades aborígenes (Madrid: Centro de Estudios Políticos y Constitucionales, 2002); Claudia García "Interacción étnica y diplomacia de fronteras en el reino miskitu a fines del siglo XVIII", Anuario de Estudios Americanos, vol. 56, n. ${ }^{\circ} 1$ (1999): 95-121; Carlos Lázaro Ávila, "Conquista, control y convicción: el papel de los parlamentos indígenas en México, el Chaco y Norte América", Revista de Indias, vol. 59, n. 217 (1999): 645-673.

$6 \quad$ Alison Brysk, De la tribu a la aldea global: Derechos de los pueblos indigenas, redes transnacionales y relaciones internacionales en América Latina (Barcelona: Ediciones Bellaterra, 2009). 
recursos técnicos y financieros provenientes de la cooperación internacional y los propios. La concatenación de los diferentes actores (muchas veces como redes de defensa) hicieron posible la transmisión de las energías sociales de lo local hacia lo global en distintos grados de velocidad e intensidad ${ }^{7}$.

La actividad diplomática indígena incursionó en organismos internacionales — como en las Naciones Unidas - para influir y transformar la acción de representantes estatales o funcionarios con poder de decisión sobre políticas y programas que son de vital importancia para sus demandas y reivindicaciones. Resultados visibles de influencia fueron la Declaración de la Naciones Unidas sobre los Derechos de los Pueblos Indígenas (2007), el Día Internacional de los Pueblos Indígenas, el Año Internacional de las Poblaciones Indígenas del Mundo (1993), la creación del relator especial sobre los derechos de los pueblos indígenas, el primero y segundo Decenio Internacional de las Poblaciones Indígenas del Mundo (1995-2004 y 2005-2015), en el que también incidieron las mujeres indígenas.

El papel de las mujeres indígenas es central y destacado en escenarios internacionales, como en la Plataforma Beijing (1995), con sus demandas en el estudio sobre la participación política de las mujeres indígenas de Naciones Unidas (2013), así como la exigencia del análisis preparado de la secretaría del Foro Permanente sobre las Mujeres Indígenas (2009) y la declaratoria del 5 de septiembre como el Día Internacional de la Mujer Indígena, creado desde 1983 —entre los más destacados—, que permitió movilizar sus causas hacia sus Estados y frente a actores poderosos, como empresas transnacionales.

Las investigaciones sobre la incursión diplomática indígena en escenarios oficiales internacionales han ganado espacio en la literatura especializada. Sin embargo, la actividad diplomática indígena continental autoconvocada por líderes y lideresas de la región tiene menos volúmenes, pese a su valiosa contribución a la agenda política latinoamericana. A diferencia de investigaciones realizadas en Australia, Nueva Zelanda, Estados Unidos y Canadá, por nombrar los emblemáticos, en América Latina ${ }^{8}$ aún existe una deuda importante. Contribuir a llenar ese vacío es el propósito de presentar una parte de los resultados de dos investigaciones desarrolladas por los autores sobre la diplomacia indígena.

Sanjeev Khagram, James V. Riker y Kathryn Sikkink, Restructuring world politics: Transnational social movements, networks, and norms (Minneapolis: University of Minnesota Press, 2002).

8 José Bengoa, La emergencia indígena en América Latina (Santiago: Fondo de Cultura Económica, 2007). 


\section{Planteamiento del problema y estrategia metodológica}

Evocando las cumbres interestatales, líderes y lideresas indígenas convocaron las organizaciones indígenas de diferentes países para realizar conjuntamente análisis coyunturales, fijar agendas continentales, establecer temáticas prioritarias, renovar pactos y declarar sus demandas y propuestas en documentos oficiales en clave de horizonte político. En este contexto, el planteamiento de la investigación fue considerar: ¿cuáles fueron las dimensiones políticas, funcionales y simbólico/ culturales de la diplomacia indígena en los encuentros y cumbres continentales de pueblos, naciones y mujeres indígenas que le han dado forma y contenido a su agenda política en América Latina?

Siguiendo la reconstrucción histórica de los encuentros y cumbres ancestrales desde 1990 hasta 2019, se buscó identificar las dimensiones político-culturales y funcionales de cada una de ellas y agruparlas en tendencias o aspectos que permitieran analizar (desagregar) y comprender el significado evolutivo en las últimas tres décadas. Fueron usadas herramientas de recolección y análisis de información cualitativos para identificar y reorganizar las sinergias de los eventos de estudio (cumbres y encuentros), con base en patrones de relación implícitos y explícitos. La recolección de la información fue realizada por medio de la observación estructurada (participante), entrevistas semiestructuradas y acopio de información documental especializada de la siguiente manera:

a. La recolección de información documental se realizó en diferentes bases de datos físicas y digitales de bibliotecas y archivos personales en Bolivia, Ecuador, Colombia y México, entre 2012 y 2019. Se realizaron varios viajes a dichos países para este propósito.

b. Con las visitas a los países mencionados, fueron aplicadas entrevistas a decenas de lideresas y líderes indígenas que ocupaban cargos en organizaciones indígenas nacionales y regionales, así como dirigentes indígenas con cargos en el Estado (Bolivia, fundamentalmente). Las decenas de entrevistas semiestructuradas fueron capturadas en audiovisual y audio, y en ellas básicamente se exploraron los puntos neurálgicos de la agenda política y la manera como la dimensión espiritual ancestral influía en su orientación.

c. El proceso de participación tuvo lugar en varios escenarios: en la II Cumbre de Mujeres Indígenas y en la V Cumbre Continental de Pueblos Indígenas del Abya 
Yala del 2013, en el resguardo indígena de La María (Cauca), Colombia; en la II Cumbre Regional Amazónica realizada en 2013 en Villavicencio, Colombia; en el Encuentro de Mujeres que Lucha, realizada del 8 al 10 de marzo de 2018 en Chiapas, México; en el I Encuentro Nacional de Mujeres hacia la Agenda de la Despatriarcalización y Descolonización para Vivir Bien 2020-2030, celebrada en octubre de 2018 en Cochabamba, Bolivia; y en la Mesa Permanente de Concertación Nacional de los Pueblos Indígenas de 2019, en Bogotá, Colombia.

El análisis de la información permitió identificar dos tendencias principales que son "patrones fuerza" que caracterizan a las cumbres y encuentros. La primera dimensión es la política-étnica, en la que se encuentran tres principales hallazgos que se produjeron durante el encuentro diplomático: a) el horizonte político indígena latinoamericano evolucionó hacia la concepción de la 'toma del poder'; b) el cambio de milenio se concibió como una señal del tiempo cíclico para la recuperación del territorio ancestral, tomando como referencia la autodeterminación; c) la espiritualidad fue la inspiración y guía de las luchas ancestrales. La segunda dimensión fue la política de género desarrollada por las mujeres indígenas en los encuentros diplomáticos, en los que reclamaron su participación en los procesos de dirección indígena y como representantes de la agencia de la política ancestral (tabla 1).

Tabla 1. Historia de Encuentros y Cumbres Continentales de Pueblos Indígenas de Abya Yala /América (1990-2019)

\begin{tabular}{|l|l|l|l|l|}
\hline \multicolumn{1}{|c|}{ Nombre de la cumbre } & Año & $\begin{array}{l}\text { Nombre de la } \\
\text { Declaración }\end{array}$ & Temas y palabras clave & Lugar \\
\hline $\begin{array}{l}\text { I Encuentro Continental de } \\
\text { Pueblos Indios }\end{array}$ & 1990 & $\begin{array}{l}\text { Declaración de } \\
\text { Quito }\end{array}$ & $\begin{array}{l}\text { Autodeterminación } \\
\text { V Centenario } \\
\text { Autogobierno }\end{array}$ & Quito, Ecuador \\
\hline $\begin{array}{l}\text { II Encuentro Continental de la } \\
\text { Campaña 500 años de Resisten- } \\
\text { cia Indígena, Negra y Popular }\end{array}$ & 1991 & $\begin{array}{l}\text { Declaración de } \\
\text { Xelajú }\end{array}$ & $\begin{array}{l}\text { V Centenario } \\
\text { Alianzas populares } \\
\text { Antineoliberalismo }\end{array}$ & Guatemala \\
\hline $\begin{array}{l}\text { III Encuentro Continental de la } \\
\text { Resistencia Indígena, Negra y } \\
\text { Popular }\end{array}$ & 1992 & $\begin{array}{l}\text { Declaración de } \\
\text { Mangua }\end{array}$ & $\begin{array}{l}\text { V Centenario } \\
\text { Alianzas populares } \\
\text { Antineoliberalismo }\end{array}$ & $\begin{array}{l}\text { Managua, } \\
\text { Nicaragua }\end{array}$ \\
\hline $\begin{array}{l}\text { II Encuentro Continental de Pue- } \\
\text { blos Indígenas }\end{array}$ & 1993 & $\begin{array}{l}\text { Declaración de } \\
\text { Temoaya }\end{array}$ & $\begin{array}{l}\text { Espiritualidad y ritualidad } \\
\text { Modelo de desarrollo }\end{array}$ & $\begin{array}{l}\text { Temoaya, } \\
\text { México }\end{array}$ \\
\hline I Cumbre Continental Indígena & 2000 & $\begin{array}{l}\text { Declaración de } \\
\text { Teotihuacán }\end{array}$ & $\begin{array}{l}\text { Libre determinación } \\
\text { Estados pluriétnicos } \\
\text { Cambios en el movimiento } \\
\text { indígena }\end{array}$ & $\begin{array}{l}\text { Teotihuacán, } \\
\text { México }\end{array}$ \\
\hline
\end{tabular}




\begin{tabular}{|c|c|c|c|c|}
\hline Nombre de la cumbre & Año & $\begin{array}{l}\text { Nombre de la } \\
\text { Declaración }\end{array}$ & Temas y palabras clave & Lugar \\
\hline $\begin{array}{l}\text { II Cumbre Continental de Pue- } \\
\text { blos y Nacionalidades Indígenas } \\
\text { del Abya Yala }\end{array}$ & 2004 & $\begin{array}{l}\text { Declaración de } \\
\text { Kito }\end{array}$ & $\begin{array}{l}\text { Foro Social Mundial } \\
\text { Derechos de los pueblos } \\
\text { indígenas } \\
\text { Ofensiva neoliberal }\end{array}$ & Quito, Ecuador \\
\hline $\begin{array}{l}\text { Cumbre Continental de Pueblos } \\
\text { y Organizaciones Indigenas } \\
\text { Territorio Mapuche }\end{array}$ & 2005 & $\begin{array}{l}\text { Declaración de } \\
\text { la Cumbre Con- } \\
\text { tinental de Pue- } \\
\text { blos y Organiza- } \\
\text { ciones Indígenas } \\
\text { en Territorio } \\
\text { Mapuche, Mar } \\
\text { del Plata }\end{array}$ & $\begin{array}{l}\text { Globalización, desarrollo y } \\
\text { pluralismo jurídico }\end{array}$ & $\begin{array}{l}\text { Mar de Plata, } \\
\text { Argentina }\end{array}$ \\
\hline $\begin{array}{l}\text { I Encuentro Continental de Pue- } \\
\text { blos y Nacionalidades Indigenas } \\
\text { del Abya Yala "De la resistencia } \\
\text { al poder" }\end{array}$ & 2006 & $\begin{array}{l}\text { Declaración de } \\
\text { La Paz }\end{array}$ & $\begin{array}{l}\text { Refundación del Estado } \\
\text { Plurinacionalidad }\end{array}$ & La Paz, Bolivia \\
\hline $\begin{array}{l}\text { III Cumbre Continental de Pue- } \\
\text { blos y Nacionalidades Indigenas } \\
\text { de Abya Yala "De la resistencia } \\
\text { al poder" }\end{array}$ & 2007 & $\begin{array}{l}\text { Declaración de } \\
\text { Iximche }\end{array}$ & $\begin{array}{l}\text { Acceso y refundación del } \\
\text { Estado } \\
\text { Plurinacionalidad } \\
\text { Liderazgos de mujeres y } \\
\text { jóvenes }\end{array}$ & $\begin{array}{l}\text { Ixemche, } \\
\text { Guatemala }\end{array}$ \\
\hline $\begin{array}{l}\text { IV Cumbre Continental de Pue- } \\
\text { blos y Nacionalidades Indigenas } \\
\text { del Abya Yala, "ipor el Sumak } \\
\text { Kawsay! BBuen vivir!" }\end{array}$ & 2009 & $\begin{array}{l}\text { Declaración de } \\
\text { Mama } \\
\text { Quta Titikaka }\end{array}$ & $\begin{array}{l}\text { Buen vivir } \\
\text { Estados plurinacionales } \\
\text { comunitarios }\end{array}$ & Puno, Perú \\
\hline $\begin{array}{l}\text { I Cumbre Regional Amazónica } \\
\text { Saberes Ancestrales, Pueblos y } \\
\text { Vida Plena en Armonía con los } \\
\text { Bosques }\end{array}$ & 2011 & $\begin{array}{l}\text { Declaración de la } \\
\text { I Cumbre Regio- } \\
\text { nal Amazónica } \\
\text { Saberes Ances- } \\
\text { trales, Pueblos } \\
\text { y Vida Plena en } \\
\text { Armonía con los } \\
\text { Bosques }\end{array}$ & $\begin{array}{l}\text { Crisis climática y ambiental } \\
\text { Legislación y política } \\
\text { pública frente a los bosques } \\
\text { y territorios amazónicos }\end{array}$ & Manaos, Brasil \\
\hline $\begin{array}{l}\text { II Cumbre Continental de Muje- } \\
\text { res Indigenas de Abya Yala y V } \\
\text { Cumbre Continental de Pueblos } \\
\text { Indigenas del Abya Yala }\end{array}$ & 2013 & $\begin{array}{l}\text { Declaración } \\
\text { de La María } \\
\text { Piendamó }\end{array}$ & $\begin{array}{l}\text { Diplomacia de los pueblos } \\
\text { Autodeterminación de los } \\
\text { pueblos } \\
\text { Buen vivir, paradigma } \\
\text { político } \\
\text { Conferencia Mundial de los } \\
\text { Pueblos Indígenas } \\
\end{array}$ & $\begin{array}{l}\text { La María, Pien- } \\
\text { damó (Cauca), } \\
\text { Colombia }\end{array}$ \\
\hline II Cumbre Regional Amazónica & 2013 & $\begin{array}{l}\text { Juntos por la } \\
\text { defensa de la } \\
\text { Amazonía y un } \\
\text { planeta vivo }\end{array}$ & $\begin{array}{l}\text { Desarrollo, megaproyectos, } \\
\text { biodiversidad, agenda inter- } \\
\text { nacional de influencia }\end{array}$ & $\begin{array}{l}\text { Villavicencio, } \\
\text { Colombia }\end{array}$ \\
\hline
\end{tabular}




\begin{tabular}{|l|c|l|l|l|}
\hline \multicolumn{1}{|c|}{ Nombre de la cumbre } & Año & $\begin{array}{l}\text { Nombre de la } \\
\text { Declaración }\end{array}$ & Temas y palabras clave & \multicolumn{1}{|c|}{ Lugar } \\
\hline $\begin{array}{l}\text { I Encuentro de Mujeres que } \\
\text { Luchan }\end{array}$ & 2018 & $\begin{array}{l}\text { Palabras de las } \\
\text { mujeres zapatis- } \\
\text { tas en la clausura }\end{array}$ & $\begin{array}{l}\text { Defensa del territorio, estra- } \\
\text { tegias colectivas de lucha, } \\
\text { denuncia de la violencia } \\
\text { patriarcal }\end{array}$ & $\begin{array}{l}\text { Caracol More- } \\
\text { lia, Altamira, } \\
\text { Chiapas }\end{array}$ \\
\hline $\begin{array}{l}\text { I Encuentro Nacional de Mujeres } \\
\text { hacia la Agenda de la Despa- } \\
\text { triarcalización y Descolonización } \\
\text { para Vivir Bien 2020-2030 }\end{array}$ & 2018 & $\begin{array}{l}\text { Agenda de Des- } \\
\text { patriarcalización } \\
\text { y Descoloniza- } \\
\text { ción para Vivir } \\
\text { Bien 2020-2030 }\end{array}$ & $\begin{array}{l}\text { Políticas de despatriarcaliza- } \\
\text { ción, buen vivir, reelección } \\
\text { de Evo Morales Ayma }\end{array}$ & $\begin{array}{l}\text { Cochabamba, } \\
\text { Bolivia }\end{array}$ \\
\hline
\end{tabular}

Fuente: Elaboración propia.

\section{La política ancestral y las cumbres indígenas latinoamericanas}

La diplomacia lleva mensajes políticos que quedan consignados en declaraciones de las cumbres, en los que se fija el horizonte político y se hace un llamado a la comunidad global para buscar su apoyo y poner en conocimientos los temas tratados. Las dimensiones políticas de las cumbres son el conjunto de reivindicaciones, exigencias, solicitudes y/o necesidades construidas por los representantes indígenas. Usualmente, se convierten en plataformas políticas continentales con objetivos políticos — de cambio o reforma - que buscan agrupar la diversidad política y cultural de pueblos, naciones y organizaciones indígenas.

Las gramáticas políticas se fundamentan, además, en la interpretación del espaciotiempo contemporáneo, sagrado, prehispánico y de larga resistencia, que da sentido a la identidad étnica continental y se articulan a dimensiones espirituales provenientes de sus cosmovisiones, saberes y prácticas ancestrales que invocan y convocan a entidades espirituales (fuego, agua, montañas, animales, ancestros) para que sus actuaciones sean guiadas y acompañadas. El encuentro diplomático integra reivindicaciones políticas, concepciones autocomprensivas del espacio-tiempo de los pueblos y evocación espiritual, como se verá a continuación.

\subsection{Gramáticas políticas: 'De la resistencia a la toma del poder'}

En la década de los noventa, las reuniones continentales indígenas fueron llamadas encuentros, pero progresivamente tomaron la connotación de cumbres, al ofrecer un estatus internacional a los pueblos y naciones indígenas al mismo nivel de las 
relaciones interestatales. Esta adaptación creativa tuvo el propósito de "delinear objetivos, estrategias, plataformas de acción, refrendar y modificar agendas y reafirmar la unidad alrededor del proyecto político indígena continental", en el marco de una interpretación política de autodeterminación y autonomía. Los encuentros y cumbres surgieron como mecanismos estratégicos continentales motivados por el fortalecimiento de los movimientos indígenas locales y en medio de un convulsionado ambiente por la implementación del neoliberalismo en Latinoamérica.

La intensidad de los encuentros diplomáticos de principios de la década de los noventa fue motivada por la disputa del significado de los 500 años de la llegada de Cristóbal Colón. La discusión fue iniciada a mediados de los años ochenta en foros internacionales sobre los derechos humanos de los pueblos indios y en encuentros campesinos-indígenas, para confrontar las celebraciones oficiales iberoamericanas sobre el V Centenario del 'Descubrimiento de América'. La consigna de "500 años de resistencia" se convirtió en el código de integración continental que atrajo representantes y observadores de Europa y África. La Campaña Continental 500 años de Resistencia Indígena, Negra y Popular fue el pilar de las cumbres y encuentros posteriores, al demostrar la importancia de la unidad continental.

El Encuentro Continental de Quito en 1990 puso el acento en las nociones de resistencia, al explicitar, según la declaración, lo siguiente: "en nuestra estrategia general de lucha, consideramos prioritario exigir las modificaciones de fondo, que permitan el ejercicio pleno de la autodeterminación a través de gobiernos propios de los pueblos indios y del control de nuestros territorios". La autodeterminación puso de manifiesto una discusión de carácter internacional, al hablar sobre las soberanías estatales en una doble dimensión: como crítica al imperialismo y neocolonialismo ejercidos en correlaciones de fuerza de carácter global y local, y como una opción política para la supervivencia y el desarrollo de los pueblos indígenas sin intermediación y paternalismos.

Entre 1993 y 2005 fue promovida la necesidad de intensificar el impulso diplomático para generar formas de unidad continental. La agenda diplomática se caracterizó por las gramáticas de resistencia de oposición al Estado criollo y los actores transnacionales del desarrollo neoliberal que se venían apoderando de sus territorios. Este periodo de intensidad diplomática se caracterizó por la consolidación de la identidad

Araceli Burguete, "Cumbres Indígenas en América Latina: Cambios y continuidades en una tradición política. A propósito de la III Cumbre Continental Indígena en Guatemala", 2007, consultado marzo, 2020. www. nacionmulticultural.unam.mx/movimientosindigenas/docs/decl_278.pdf 
política continental, entendida como un conjunto de creencias y sentimientos de solidaridad de una vivencia histórica compartida. Durante la década de los noventa, la agenda diplomática giró sobre la "resistencia contra los sistemas de dominación existentes en el continente", "fortalecer las organizaciones y trazar lineamientos claros" (1990), aspiraciones que deberían traducirse en "la autodeterminación como derecho inalienable e imprescindible de los pueblos indígenas" (1990), y en aspectos más específicos, como la defensa y articulación de la unidad política en torno a la espiritualidad y la tradición (1993), la defensa de la Madre Tierra y el control sobre el territorio y sus recursos (1993); el reconocimiento jurídico de la autonomía indígena, el rol e importancia de la mujer, y la defensa de la educación basada en la filosofía de los pueblos indígenas (1993).

Marcados por el acontecer internacional de las Naciones Unidas, con el borrador de la Declaración de los Derechos de los Pueblos Indígenas surgieron manifestaciones explícitas para que se materializara su aprobación y se sostuvieran nociones importantes, como "pueblos" y "autodeterminación". Igualmente, con el nacimiento de documentos clave provenientes de la Cumbre de Río (1992), se exigió la participación efectiva de los pueblos indígenas en el Convenio sobre la Diversidad Biológica, y se enfatizó en la denuncia de la "política financiera impuesta por el Fondo Monetario Internacional, el Banco Mundial y el Banco Interamericano de Desarrollo, con la complicidad de los gobiernos" (1993), y la materialización de la Declaración Universal de los Derechos Humanos para los pueblos indígenas. La década de los noventa dejó experiencias interesantes que demostraron la capacidad de los pueblos indígenas para enfrentar con eficacia la presión política estatal y su especial participación en el ámbito internacional.

En el segundo milenio, los encuentros y cumbres continentales indígenas tuvieron sus referentes en el movimiento indígena ecuatoriano y el nacimiento del Ejército Zapatista de Liberación Nacional (EZLN) en el sur de México. En el ámbito nacional, las constituciones latinoamericanas incluyeron la perspectiva multiculturalista; finalmente, la democracia se había establecido. En el ámbito internacional, se vio con decepción la falta de voluntad de los Estados para aprobar la Declaración de los Derechos de los Pueblos de la ONU, debido a la marcada influencia de las políticas de desarrollo del Banco Mundial (BM), el Fondo Monetario Internacional (FMI) y el Banco Interamericano de Desarrollo (BID) sobre sus territorios y la incursión 
cada vez más fuerte de las empresas transnacionales que llevan a la destrucción de los sistemas ecológicos y sus pueblos ${ }^{10}$.

Ante estas nuevas expresiones del capitalismo, las manifestaciones políticas de las cumbres y encuentros reafirmaron sus críticas y demandas. Lo que resulta más interesante, a partir de entonces, es la forma como las gramáticas políticas enunciaron desde una visión ancestral sus apuestas de autodeterminación y autonomía. En la II Cumbre Continental de los Pueblos y Nacionalidades Indígenas de Abya Yala de 2004, en Quito, aparece una resignación de los códigos políticos influenciados por el discurso político andino, al señalar: "Somos pueblos originarios de Abya Yala. Nuestros antepasados, nuestros abuelos nos enseñaron a amar y venerar nuestra fecunda Pacha Mama". Abya Yala es una reapropiación sobre la forma de nombrar el territorio americano desde la lengua kuna, y el término de Pachamama es una expresión en quechua para nombrar a la Madre Tierra. Además, aparece la noción de Estado plurinacional del movimiento indígena ecuatoriano, que después sería prácticamente una forma de interpretación política de la autodeterminación y la autonomía continental. Además, la cumbre de 2004 ofreció su apoyo a Cuba y Venezuela, y en especial al presidente Hugo Chávez Frías: "Solidarizarnos con el pueblo de Venezuela y el presidente Hugo Chávez" y "Solidarizarnos con el pueblo Cubano por su permanente lucha antiimperialista".

Los intensos levantamientos de los movimientos aymaraquechuas en Bolivia (2000, 2001 y 2003) demostraron la capacidad del movimiento indígena para derrotar a los gobiernos neoliberales y asumir la vanguardia política. El triunfo electoral de Evo Morales (2006) encarnó el resultado de una lucha indígena quechuaymara que pasaba de la resistencia al ejercicio del poder. La nueva gramática política se dirigió a "la refundación de los Estados-nación y la construcción de los Estados plurinacionales y sociedades interculturales a través de las Asambleas Constituyentes con representación directa de los pueblos y nacionalidades indígenas"11.

Revisando las declaraciones a partir de 2006, se encontraron los lemas "De la resistencia al poder” (2006, 2007), y “¡Por el Sumak Kawsay! ¡Buen Vivir!” (2009), con el claro

10 Tenemos un desarrollo de megaproyectos, como la Integración de Infraestructura Regional Sudamericana (IIRSA); el Plan Puebla Panamá (PPP); el Plan Patriota; el Plan Colombia; el Plan Dignidad; el Plan Andino, y la creación de bases militares, así como al establecimiento del Área de Libre Comercio de las Américas (ALCA) y los tratados de libre comercio (TLC), impulsados en el marco de la Organización Mundial del Comercio (OMC).

11 III Cumbre Continental de Pueblos y Nacionalidades Indígenas de Abya Yala "De la resistencia al poder", Guatemala, 26 al 30 de marzo de 2007 
objetivo de "construir Estados Plurinacionales Comunitarios, que se fundamenten en el autogobierno, la libre determinación de los pueblos, la reconstitución de los territorios y naciones originarias" (2009). Las agendas diplomáticas incorporaron relatos de refundación civilizacional en ámbitos políticos (Estado plurinacional), sociales (sociedades interculturales), económicos (el buen vivir) ${ }^{12}$ y culturales (una refundación de las relaciones humanas con la Pachamama o Madre Tierra).

\subsection{El tiempo ha llegado: es hora de recuperar el territorio ancestral}

Las cumbres y encuentros discutieron la concepción hegemónica del espaciotiempo del Estado nación criollo. Los representantes indígenas insistieron en que el sustento cultural del Estado nacional ha beneficiado a las élites y excluido a los pueblos originarios. El acento en esta discusión estuvo presente en los encuentros continentales que afirmaron que bajo la invención de la soberanía estatal existe la soberanía ancestral.

De la misma manera que el Estado legitima su pasado, las declaraciones indígenas evocaron tiempos y espacios propios: "Desde el corazón del mundo, en el lugar del sol recto" o la Cumbre Indigena Continental reunida al pie del Centro Ceremonial de Teotihuacán, "autoconvocados y reunidos en Iximche, los días oxlajuj Aq’abal, trece fuerzas del espíritu del amanecer, 26 al kají kej, cuatro fuerzas del espíritu del Venado", "Reunidos en la Paqarina Mayor de Lago Mama Qota Titikaka". La gramática diplomática evocó la sacralidad del lugar, el ordenamiento propio que emana de leyes originarias y las concepciones de la ontología de cada pueblo: "Esta perspectiva de espacio sagrado corresponde a la realidad terrenal, desde donde se vinculan con otros mundos, el mundo de la forma se alimenta de las deidades y del inframundo para mantener el equilibrio natural"13.

La recuperación del territorio como un espacio-tiempo sagrado vislumbró una ruptura con el territorio-estatal que dio sentido a la diplomacia moderna. Al incluir las dimensiones políticas y cósmicas sobre los espacios, hubo una reinscripción

12 Las cumbres y encuentros indígenas adoptaron la noción de buen vivir en varios sentidos. Primero, como una crítica al impulso ontológico de la civilización occidental, sus instituciones, sus discursos y sus prácticas, y como propuesta para una refundación de la historia planetaria. Segundo, como una crítica al modelo de desarrollo capitalista sustentado en el crecimiento económico, el bienestar material, el consumo, el mercado, la mercantilización de la naturaleza y las formas de explotación, que conllevan riesgo planetario global. Y tercero, como la implementación de modelos propios de gobierno, marcos jurídicos y de justicia, educativos, económicos, productivos y organizativos.

13 Jair Zapata Torres, "Espacio y territorio sagrado. Lógica del ordenamiento territorial indígena". línea, vol. 20 (2007) https://issuu.com/carloslopezpiedrahita/docs/libroespacioyterritorio24_03_10 
de la historia política del lugar y, por lo tanto, de lo que representa la diplomacia indígena. La concepción del espacio-tiempo sagrado evocó la fuerza territorial local, pero no se agotó en sí misma. La evocación contemporánea de estas gramáticas tuvo un triple contenido espacio-temporal: un tiempo prehispánico, el inicio de un proceso de resistencia de larga duración de más de quinientos años, y las actuales luchas contra la globalización y el neoliberalismo.

Con el tiempo prehispánico, afirmaron: "somos herederos de las civilizaciones originarias que alcanzaron los más [altos] niveles de conocimientos y de organización comunitaria"14, basados en "espiritualidad, comunidad, creatividad y tecnologías que sostienen el medio ambiente y soportan los aspectos esenciales de la vida en un permanente movimiento del tiempo y el espacio"15, y que generan así formas propias de gobierno y leyes, con las que "los pueblos indígenas han intentado construir relaciones colectivas integrales"16. El tiempo-espacio de resistencia fueron los quinientos años de la modernidad/capitalista, hasta lo que consideran como "la profunda crisis de la civilización occidental capitalista"17. En América, el tiempo-espacio de crisis se inscribe en las nociones de Pachakutik como noción de cambio: "retorno del tiempo, de la vida, del equilibrio, de la armonía", que evoca un "tiempo de transición de un sol a otro, un tiempo de reordenamiento"18, tal como se lee en las declaraciones: "nosotros los pueblos y nacionalidades indígenas de Abya Yala estamos ante el resurgimiento del Pachakutik"19. Por su parte, el espacio-tiempo coyuntural, usó la celebración de las cumbres y encuentros en los países donde se necesitaba apoyo político, tal como Bolivia, para respaldar a Evo Morales, o Colombia, para exigir la paz en los territorios indígenas. En resumen, la recuperación del territorio y la interpretación de la acción política como un espacio-tiempo sagrado vislumbró una ruptura con el territorio-estatal y evocó un proyecto sociopolítico futuro con raíces en la ancestralidad.

\footnotetext{
14 "Declaración de Managua (12 de octubre de 1992)". En Voces Indias y V Centenario, coordinado por Sergio Sarmiento Silva (México D. F.: Instituto Nacional de Antropología, 1998).

15 Gregory Cajete, Native science: Natural laws of interdependence (Nuevo México: Clear Light Pub, 2000), 3.

16 "Consejo Mundial de Pueblos Indígenas, La Cumbre y la bases". En Sarmiento, Voces Indias y V Centenario.

17 "Declaración de Mama Quta Titikaka en el marco de la IV Cumbre de Pueblos de Abya Yaya", ¡12 al 16 de octubre Movilización global en Defensa de la Madre Tierra y los Pueblos! (2009). movimientos.org/es/ defensamadretierra/show_text.php3\%3Fkey\%3D15515

18 Fernando Huanacuni Mamani, "Cosmovisión Andina, Parte 3. Vivir Bien/Buen Vivir". En Conjunto de visiones sobre la postmodernidad. Ciclo de conferencias, editado por Fernando Huanacuni Mamani (La Paz: Publicaciones Casa de la Libertad, 2012), 70.

19 "Declaración final de la III Cumbre Continental de Pueblos y Nacionalidades Indígenas de Abya Yala", De la Resistencia al Poder, Iximche' Iximulew, Guatemala 26 al 30 de marzo de 2007.
} 


\subsection{La política guiada por la espiritualidad}

Al inicio de la II Cumbre Continental de Mujeres Indígenas del Abya Yala y de la V Cumbre Continental de los Pueblos del Abya Yala (2013), los tatas y nanas encendieron el primer fuego ritual para pedir permiso y convocaron la presencia del corazón del cielo, de la Madre Tierra, del Sol y la Luna, y al espíritu del Padre Fuego, para que acompañaran, dieran fuerza y sabiduría a los participantes de la Cumbre. Una vez fue encendido el fuego, continuaron cuidándolo día y noche durante todos los días del encuentro diplomático. Alrededor, se elaboró la chakana, que representa los cuatro puntos cardinales de donde provenían todos los pueblos indígenas. En el centro se dibujó un espiral en forma de caracol formado por hojas y semillas, frutas, panes y objetos que los participantes iban aportando para que el fuego estuviera protegido. Cada día, los abuelos y abuelas realizaron el ritual de limpieza y armonización para alejar espíritus y fuerzas negativas que impidieran el equilibrio con la Pachamama y entorpecieran los propósitos del encuentro diplomático. Con esta primera mediación, el escenario quedó adecuado para abordar la agenda política y encarar las negociaciones, diálogos, reflexiones y debates bajo la guía permanente del Fuego Sagrado.

Costas Constantinou afirma que la espiritualidad en la diplomacia ha sido históricamente determinante. En el pasado, ha transformado relaciones hostiles o potencialmente hostiles, por mediaciones etnorreligiosas de reconocimiento y aprendizaje de la otredad, en las que el reino espiritual ha desempeñado un papel determinante para la sostenibilidad pacífica de las relaciones entre diferentes entidades políticas ${ }^{20}$. Recuerda que tradiciones diplomáticas que han perseguido 'el mejor estar' de las comunidades parten de dimensiones espirituales, como el caso de los nativos americanos que fumaron la 'pipa de la paz' como ritual para regular relaciones intertribales y preparar el encuentro diplomático, un medio que sirve para "negociar" verticalmente con sus dioses y antepasados, y también introspectivamente (entre las entidades tribales), al invitar a los poderes espirituales para mejorar la perspectiva de sus relaciones horizontales con los demás.

Gregory Cajete ${ }^{21}$ afirma que, tradicionalmente, los pueblos indígenas han comprendido que los pactos entre los humanos deben celebrarse en coparticipación ritual con las fuentes de vida de la tierra y con las entidades naturales del lugar. Como lo afirma Roy Rappaport: "el ritual es un modo especialmente adecuado, incluso único, para

20 Costas, "On homo-diplomacy".

21 Cajete, Native science. 
la transmisión de ciertos mensajes y de cierto tipo de información"22. Los rituales tienen su propia teleología, sus fines explícitos ${ }^{23}$, se encuentran estrechamente vinculados con las circunstancias en las cuales se celebra y tienen una funcionalidad comunicativa entre los participantes y con las entidades espirituales encarnadas en formas materiales e inmateriales.

El ritual de "encendido del Fuego Sagrado" en encuentros y cumbres continentales se convirtió en una tradicional forma de interpretación de la alteridad humana y cósmica, y en el requisito indispensable para el inicio y cierre del encuentro diplomático ancestral contemporáneo. El poder del Fuego Sagrado abrió y adecuó la actividad diplomática para que pudiera desarrollarse en armonía y equilibrio, su copresencia durante la mediación fue tomada como indispensable para el éxito de las cumbres, pues tiene el poder de adecuar la voluntad y sapiencia de los participantes, y convocar a las fuerzas cósmicas para que animen las buenas decisiones, medien con armonía los posibles conflictos y mantengan el compromiso de los pueblos indígenas y sus organizaciones con la unidad para la lucha:

El Fuego Sagrado nos ilumina todavía, dejando huellas de nuestro sendero sagrado y recordándonos la historia verdadera de nuestro movimiento indígena continental. Encarnado de acuerdo con la ética milenaria de nuestros Pueblos, y con nuestra propia Ley Internacional Indígena, estos compromisos mutuos al nivel continente siguen vigentes por medio del Tratado de Teotihuacán, la constitución de la Confederación Continental del Águila y el Cóndor. ${ }^{24}$

El encendido del Fuego Sagrado representó el sostenimiento de los encuentros diplomáticos entre los pueblos indígenas del norte y del sur de América, y llevó mensajes a los asistentes de las cumbres, por medio de la interpretación de las autoridades tradicionales, que fueron como "embajadores" entre los mundos metafísico y humano ${ }^{25}$. Finalmente, fue una práctica reivindicativa, en la que el

22 Roy Rappaport, Ritual y religión en la formación de la humanidad (Madrid: Akal Editores, 2001), 65.

23 Víctor Turner, La selva de los símbolos (México D.F: Siglo Veintiuno, 1999), 35.

24 Tupac Enrique Acosta, "Propuesta al Caucus de Pueblos Indígenas" (México Foro Permanente para las Cuestiones Indígenas-ONU, sexto periodo de sesiones, mayo 14-25, 2007), consultado agosto 13, 2019. www.indiosonline.net/pueblos_indigenas/

25 Un particular trabajo que explica la autoridad de los mayores para interpretar la Ley de Origen: Julia SuárezKrabbe, "Identidad y ser. Un análisis de ausencias y emergencias con los mamos de la Sierra Nevada de Santa Marta". En Formas-otras. Saber, nombrar, narrar, hacer, organizado por Gladys Lopera, 159-170 (Barcelona: CIDOB, 2011), consultado 18 septiembre, 2019. http://www.cidob.org/es/content/download/29946/356584/ version/1/file/159-170_JULIA\%20SUAREZ.pdf Ver también: Cristabell López Palomino, "El Saakhelu como parte del proceso de recuperación y fortalecimiento cultural del pueblo indígena nasa de Tierradentro, Cauca" 
ritual del encendido significó un acto de recuperación y reafirmación de la cultura ancestral, y el reconocimiento de la larga resistencia de los pueblos indígenas del continente.

\subsection{La resistencia de los pueblos será con las mujeres o no será}

Las mujeres indígenas han tenido que disputar y generar escenarios de participación por su propia fuerza dentro de los procesos organizativos indígenas continentales que han tenido una representación masculina mucho mayor. Aunque suele ser recurrente en los escenarios continentales escuchar el discurso ancestral de la complementariedad hombre-mujer, las mujeres han intervenido para afirmar que los patrones de poder que afectan a los pueblos y naciones indígenas se reproducen también dentro del movimiento en formas jerarquizadas y definidas por las mismas adecuaciones de roles de la cultura patriarcal.

Según Almudena Cabezas, el proceso continental de las mujeres indígenas estuvo marcado por dos fenómenos particulares: por un lado, un discurso indígena sensible a los temas de género, pero que no tiene una correlación coherente con la práctica política de las mujeres en los escenarios de representación y decisión; por otro, y seguramente como respuesta al primero, una práctica política de las mujeres de autoorganización y autoconvocatoria para enfrentar procesos de dominación y exclusión con los miembros de sus colectividades y con agentes externos ${ }^{26}$. Sin embargo, la incidencia de las mujeres se movió entre las demandas que se han considerado como "exclusivas" (familia, hogar, hijos), y los temas "inclusivos", como los referidos en pleno a los pueblos indígenas; para desde allí promover el debate sobre las relaciones de poder en las comunidades.

Las mujeres indígenas plantearon interrogantes muy fuertes al conjunto de los procesos organizativos, pero también alternativas. Así, formaron una dimensión importante de la diplomacia indígena de las cumbres.

La ruta de las mujeres indígenas estuvo marcada por la Cuarta Conferencia Mundial sobre la Mujer en Beijing, en 1995, en la que hicieron sentir sus posiciones, al discutir

(tesis de grado para optar el título de Antropóloga, Universidad del Cauca Facultad de Ciencias Humanas y Sociales Departamento de Antropología Popayán, 2008), 92-93.

26 Almudena Cabezas González, "Mujeres indígenas constructoras de región: desde América Latina hasta Abya Yala", Revista Internationala, vol. 4, n. ${ }^{\circ} 6$ (2012): 6. 
el énfasis excesivo en las desigualdades de género ${ }^{27}$, para resaltar el cruce de estas con otras múltiples desigualdades e injusticias que viven en sus territorios. Las discusiones abiertas en Beijing se consolidaron en la creación del Enlace Continental de Mujeres Indígenas de las Américas (ECMIA), resultado del Primer Encuentro de Mujeres Indígenas realizado en Quito, en 1995. De este espacio, además, se afirmó la necesidad de incidir en la plataforma de Beijing y en el Decenio de los Pueblos indígenas declarado por Naciones Unidas (1993-2004). Con este primer encuentro, las mujeres indígenas promovieron escenarios diplomáticos para construir una agenda común y promoción de pactos entre mujeres. En 1997 se realizó el segundo encuentro en Panamá, en el que fue posicionada la propiedad intelectual sobre los haceres y tejidos de las mujeres indígenas, y en 1999, con la reunión de diferentes mujeres del mundo, en el Taller Internacional de Mujeres Indígenas Frente al Nuevo Milenio, consolidaron la propuesta de creación de una red internacional de mujeres indígenas, que vería su nacimiento un año después en un foro que llevaría el mismo nombre de la red: Foro Internacional de Mujeres Indígenas (FIMI).

En las cumbres de indígenas, las mujeres lograron materializar un espacio para sí, el cual fue finalmente ratificado en la Tercera Cumbre Continental de Pueblos Indígenas del Abya Yala de 2007, en Guatemala. Allí se ordenó la realización de la I Cumbre de Mujeres Indígenas. Antes de su realización, las mujeres acudieron a los diferentes encuentros previos del ECMIA, tal como sucedió en Lima en el 2004 y en Quebec en el 2007, para plantear la importancia del restablecimiento del equilibrio de la Pachamama. Su postura política planteó un puente entre las demandas políticas y la dimensión espiritual de las luchas de los pueblos indígenas.

Esta posición se hizo sentir fuertemente en las cumbres que siguieron a la de Guatemala. En la I Cumbre Continental de Mujeres Indígenas del Abya Yala, realizada en Puno, Perú, en el 2009, se puso de manifiesto el aporte de las mujeres al proceso histórico indígena y se declaró la voz de nuevo pachakutik nacido de la tierra en "parto libertario'28. Esta gramática propia de las mujeres indígenas se inscribe en el relato de refundación civilizacional de la identidad étnica que da luz sobre un nuevo amanecer para los pueblos desde una discusión de género. Dicho pacto por la refundación se reafirmará en la II Cumbre Continental de Mujeres realizada

27 Tarcila Rivera, "Mujeres indígenas americanas luchando por sus derechos". En: Descolonizando el feminismo: teorías y prácticas desde los márgenes, editado por Liliana Suárez Navaz y Rosalva Aída Hernández Castillo (España: Cátedra, 2008), 331-350.

28 "Mandato de la I Cumbre Continental de Mujeres Indígenas de Abya Yala" (I Cumbre Continental de Mujeres Indígenas de Abya Yala, Puno, Perú, del 27 y 28 de mayo, 2009), consultado junio 10, 2020. https://www. latice.org/kvin/es/ilaay0907es.html 
en Colombia, en el 2013, que apuesta por la descolonización de la Madre Tierra y contra el paradigma del desarrollo capitalista, patriarcal y neoliberal vigente.

De la misma manera, otros espacios de mujeres indígenas fueron importantes, aunque fuera de las cumbres continentales. Es el caso del Encuentro de Mujeres que Luchan, realizado en el Caracol de Morelia, Chiapas, en marzo de 2018. Allí, las zapatistas lograron unificar un llamado común por la vida de las mujeres en todo el mundo, con la consigna: "Acordamos seguir vivas". Meses después se reunieron en Cochabamba, Bolivia, para reafirmar el mandato de las organizaciones por la agenda de despatriarcalización inscrito en la campaña de reelección del presidente indígena Evo Morales.

Dejaron en claro que la dirigencia indígena debe comprender las relaciones de poder en clave de interseccionalidad; es decir, uniendo las relaciones de dominación de raza, género, clase, generación, y que sería un error enfrentarlas cada una por aparte. Dicha afirmación pretendió recomponer las agendas de lucha desde una posición integral y reinterpretar las formas de resistencia y la asunción de un rol más importante de la mujer en la representación de sus pueblos y nacionalidades ${ }^{29}$.

\section{Conclusiones}

Las cumbres y encuentros indígenas probaron ser un escenario favorable a la unión de sus causas, el fortalecimiento de la identidad étnica, el favorecimiento de análisis políticos y la construcción de acuerdos conjuntos para ser llevados ante organismos internacionales, como las Naciones Unidas. Los encuentros diplomáticos permitieron recrear la identidad étnica indígena continental que, antes de los años ochenta, no era relevante para los Estados, y menos para las organizaciones de izquierda. La identidad étnica no era vista como una bandera de lucha, pues, para entonces, la explotación era sobre los obreros y campesinos, es decir, se encontraba bajo una mirada de clase. Con el progresivo encuentro de representantes indígenas en las cumbres, fue posible retomar las discusiones del indianismo, y particularmente, agrupar sus luchas por la defensa de la autodeterminación de pueblos y naciones indígenas.

29 Carmen Blanco Valer, Conferencia ofrecida en la II Cumbre de Mujeres indígenas del Abya Yala, 12 de noviembre de 2013. Audiovisual propio. 
La identidad indígena 'redescubierta' fue fortalecida en las cumbres usando la espiritualidad como una forma de integración. Los encuentros políticos indígenas colocaron en escena la recuperación de rituales y ceremonias, y lograron que la cosmovisión ancestral definiera y diera sentido a la autodeterminación y la autonomía. La concepción ontológica del mundo fue determinante para establecer puntos de la agenda política, por ejemplo: oponerse al desarrollo capitalista y proponer el buen vivir o el sumak kawsay como una alternativa a la modernidad; negar el Estado-nacional y proponer el Estado-plurinacional, con una nueva concepción de la soberanía; enfrentarse al orden patriarcal y proponer la visión de género ancestral de los opuestos complementarios. La espiritualidad evoca la relación sagrada con la Pachamama y el horizonte político entre humanos y naturaleza de forma diferente a la occidental. En este aspecto, lo político y lo espiritual van de la mano.

Los encuentros indígenas promovieron una evolución política interesante. Primero, evocaron la resistencia frente al poder de la nación criolla que los excluía, marginaba y exterminaba, en muchos casos. Posteriormente, con los hechos políticos de Bolivia y Ecuador, los representantes indígenas observaron que era posible tomar las riendas de los gobiernos y producir un Estado de muchas naciones. Esta última interpretación se tradujo en declaraciones oficiales indígenas de promover la toma del poder. El horizonte político dejó de exigir al Estado para convertirse en el Estado mismo.

La diplomacia indígena fue desde los años ochenta una de las mejores armas para atraer la solidaridad y articulación política de la sociedad global. Su práctica permitió salir de la discusión con los gobernantes de sus Estados y los llevó a tomar una dimensión política global, al establecer contactos y participación permanente en mesas internacionales, y, sobre todo, ayudó a crear una consciencia global basada en la identidad étnica como una bandera de lucha común.

\section{Referencias}

Acosta, Tupac Enrique, "Propuesta al Caucus de Pueblos Indígenas". (México Foro Permanente para las Cuestiones Indígenas-ONU, sexto periodo de sesiones, mayo 14-25, 2007), consultado agosto 13, 2015. www.indiosonline.net/ pueblos_indigenas/

Aquila, Richard. The Iroquois restoration: Iroquois diplomacy on the colonial frontier, 1701-1754.

Nebraska: Nebraska Press, 1997. 
Arévalo Robles, Gabriel Andrés. "La diplomacia indígena: un enfoque transdiplomático". Si Somos Americanos, vol. 1. n. ${ }^{\circ} 17$ (2017): 141-169. https://dx.doi.org/10.4067/ S0719-09482017000100141

Arévalo Robles, Gabriel Andrés. "Repensar la historia diplomática: diálogos, ausencias y retos para el entendimiento de la alteridad histórica mundial”. Relaciones Internacionales n. ${ }^{\circ} 37$ (2018): 121-141. doi: http://dx.doi.org/ 10.15366/relacionesinternacionales2018.37.00 Bengoa, José. La emergencia indígena en América Latina. Santiago: Fondo de Cultura Económica, 2007.

Brysk, Alison. De la tribu a la aldea global: Derechos de los pueblos indígenas, redes transnacionales y relaciones internacionales en América Latina. Barcelona: Ediciones Bellaterra, 2009. Burguete, Araceli. "Cumbres Indígenas en América Latina: Cambios y continuidades en una tradición política. A propósito de la III Cumbre Continental Indígena en Guatemala", 2007, consultado marzo, 2020. www.nacionmulticultural.unam.mx/ movimientosindigenas/docs/decl_278.pdf

Cabezas González, Almudena. "Mujeres Indígenas constructoras de región: desde América Latina hasta Abya Yala". Revista Internationala, vol. 4, n. 6 (2012): 1-22.

Cajete, Gregory. Native science: Natural laws of interdependence. Nuevo México: Clear Light Pub, 2000.

Constantinou, Costas y James Der Derian, editores. Sustainable diplomacies. New York: Palgrave MacMillan, 2010.

Constantinou, Costas. "On homo-diplomacy". Space and Culture, vol. 9, n. ${ }^{\circ} 4$ (2006): 351-364.

Constantinou, Costas. On the way to diplomacy. Minneapolis: University of Minnesota Press, 1996.

Cornago, Noé, Plural diplomacies: Normative predicaments and functional imperatives. Boston: Martinus Nijhoff Publishers, 2013.

"Declaración de Mama Quta Titikaka en el marco de la IV Cumbre de Pueblos de Abya Yaya", ¡12 al 16 de Octubre Movilización global en Defensa de la Madre Tierra y los Pueblos! (2009). movimientos.org/es/defensamadretierra/show_text.php3\%3Fkey\%3D15515 "Declaración final de la III Cumbre Continental de Pueblos y Nacionalidades Indígenas de Abya Yala", De la Resistencia al Poder, Iximche' Iximulew, Guatemala 26 al 30 de marzo de 2007.

Der Derian, James. On diplomacy: A genealogy of western estrangement. Oxford: Blackwell Publishers, 1987.

Francis, Jennings y William Fenton. The history and culture of Iroquois diplomacy: An interdisciplinary guide to the treaties of the six nations and their league. Syracuse University Press, 1995. 
García, Claudia. "Interacción étnica y diplomacia de fronteras en el reino miskitu a fines del siglo XVIII". Anuario de Estudios Americanos, vol. 56, n. 1 (1999): 95-121.

Huanacuni, Fernando. "Cosmovisión Andina, Parte 3. Vivir Bien/Buen Vivir". En Conjunto de visiones sobre la Postmodernidad. Ciclo de Conferencias, editado por Fernando Huanacuni Mamani. La Paz: Publicaciones Casa de la Libertad, 2012.

Khagram, Sanjeev, James V. Riker y Kathryn Sikkink. Restructuring world politics: Transnational social movements, networks, and norms. Minneapolis: University of Minnesota Press, 2002.

Lázaro, Carlos. "Conquista, control y convicción: el papel de los parlamentos indígenas en México, el Chaco y Norte América”. Revista de Indias, vol. 59, n. 217 (1999): 645-673.

Levaggi, Abelardo. Diplomacia hispano-indígena en las fronteras de América: Historia de los tratados entre la monarquía española y las comunidades aborígenes. Madrid: Centro de Estudios Políticos y Constitucionales, 2002.

López Palomino, Cristabell. "El Saakhelu como parte del proceso de recuperación y fortalecimiento cultural del pueblo indígena nasa de Tierradentro, Cauca" (tesis de grado, Universidad del Cauca Facultad de Ciencias Humanas y Sociales, Departamento de Antropología Popayán, 2008), 92-93

"Mandato de la I Cumbre Continental de Mujeres Indígenas de Abya Yala" (I Cumbre Continental de Mujeres Indígenas de Abya Yala, Puno, Perú, del 27 y 28 de mayo, 2009), consultado junio 10, 2020. https://www.latice.org/kvin/es/ilaay0907es.html Midtrød, Tom Arne. The memory of all ancient customs: Native American diplomacy in the colonial Hudson Valley. Cornell: University Press, 2012.

Rappaport, Roy. Ritual y religión en la formación de la humanidad. Madrid: Akal Editores, 2001. Reinaga, Fausto. Tesis india. La Paz: Ediciones PIB, 1971.

Rivera, Tarcila. "Mujeres indígenas americanas luchando por sus derechos". En Descolonizando el feminismo: teorías y prácticas desde los márgenes, editado por Liliana Suárez Navaz y Rosalva Aída Hernández Castillo, 331-350. Madrid: Cátedra, 2008.

Sarmiento, Sergio, editor. Voces Indias y V Centenario. México D. F.: Instituto Nacional de Antropología, 1998.

Suárez-Krabbe, Julia. "Identidad y ser. Un análisis de ausencias y emergencias con los mamos de la Sierra Nevada de Santa Marta”. En Formas-otras. Saber, nombrar, narrar, hacer, organizado por Gladys Lopera, 159-170. Barcelona: CIDOB, 2011. http://www. cidob.org/es/content/download/29946/356584/version/1/file/159-170_JULIA\%20 SUAREZ.pdf

Turner, Victor. La selva de los símbolos. México D. F.: Siglo XXI, 1999.

Zapata Torres, Jair. "Espacio y territorio sagrado. Lógica del ordenamiento territorial indígena". línea, vol. 20 (2007), consultado 7 de enero, 2020. https://issuu.com/ carloslopezpiedrahita/docs/libroespacioyterritorio24_03_10 